\title{
High conversion efficiency, high energy THz pulses by optical rectification in cryogenically cooled lithium niobate
}

\author{
Shu-Wei Huang ${ }^{1}$, Eduardo Granados ${ }^{1,2}$, Wenqian Ronny Huang ${ }^{1}$, Kyung-Han Hong ${ }^{1}$, Luis E. Zapata ${ }^{1}$, and \\ Franz X. Kärtner ${ }^{1,3, *}$ \\ ${ }^{1}$ Department of Electrical Engineering and Computer Science and Research Laboratory of Electronics, Massachusetts Institute of Technology, \\ Cambridge, Massachusetts 02139, USA \\ ${ }^{2}$ IKERBASQUE, Basque Foundation for Science, Spain \\ ${ }^{3}$ Center for Free-Electron Laser Science, DESY and Department of Physics, University of Hamburg, Hamburg, Germany \\ *kaertner@mit.edu
}

\begin{abstract}
We demonstrate highly efficient $\mathrm{THz}$ generation by optical rectification of near optimum pump pulses centered at $1.03 \mu \mathrm{m}$ in cryogenically cooled lithium niobate. Using a close to optimal pulse duration of $680 \mathrm{fs}$ and a pump energy of $1.2 \mathrm{~mJ}$, we report conversion efficiencies above 3.8 $\pm 0.4 \%$, which is more than an order-of-magnitude higher than previously reported. The results confirm the advantage of using cryogenic cooling of the lithium niobate crystal that significantly reduces the $\mathrm{THz}$ absorption, enabling the scaling of $\mathrm{THz}$ pulse energies to the mJ level via optical rectification.
\end{abstract}

The scope of applications that require intense and ultrafast $\mathrm{THz}$ fields has been increasing during the last years. Applications such as terahertz time-domain spectroscopy [1], the study of carrier dynamics in semiconductors [2], electric field gating of interlayer charge transport in superconductors [3], or THz assisted attosecond pulse generation [4] benefit from higher pulse energies than currently available, and so there is keen interest in scaling the peak power of the $\mathrm{THz}$ generation schemes. More recently, high peak power $\mathrm{THz}$ sources have been proposed for charged particle acceleration, undulation, deflection and spatiotemporal arbitrary manipulation too [5].

There are different methods for generating high peak field $\mathrm{THz}$ pulses. Among them, difference frequency generation (DFG) and optical rectification (OR) are the most common. Sell et al. demonstrated that it is possible to use DFG between two parametrically amplified pulse trains to generate phase locked terahertz transients with peak electric fields of $10^{8} \mathrm{MV} / \mathrm{cm}$ and center frequencies continuously tunable from 10 to $72 \mathrm{THz}$ [6]. However, such methods typically exhibit fairly low photon conversion efficiencies due to the Manley-Rowe limit and are also restricted to high $\mathrm{THz}$ frequencies approaching the mid-IR spectral region due to limitations imposed by the phase matching condition in the DFG medium, such as GaSe or $\mathrm{AgGaS}_{2}$. Optical rectification, on the other hand, has been widely implemented to generate pulses at low $\mathrm{THz}$ frequencies [7]. Because the nonlinear process can be cascaded, over $100 \%$ of photon conversion efficiency has been demonstrated $[8,9]$. Of the common nonlinear materials used for OR, ZnTe presents the problem of free carrier absorption, limiting the total efficiency [10]. Lithium niobate presents multiple advantages such as large $d_{\text {eff, }}$, high damage threshold, low $\mathrm{THz}$ absorption, and large bandgap, but it requires tilted pulse front pumping techniques to achieve 
phase matching between the IR pump and the THz wave [11]. To date, the highest $\mathrm{THz}$ pulse energies that have been reported were generated by pumping a room-temperature stoichimetric lithium niobate crystal with $100 \mathrm{~mJ}, 1.2$ ps pulses, producing sub-mJ THz pulses at relatively low efficiency (0.24\%) [5].

Recent theoretical studies have shown that OR in lithium niobate can be further improved in terms of efficiency by optimizing the pump pulse duration [12], lowering the distortions introduced by the tilted pulse front pumping optics, and reducing the photo-refractive losses in the lithium niobate crystal by cooling it down to cryogenic temperatures [13]. It has been shown that the optimum pump pulse duration is approximately $500 \mathrm{fs}$ because it maximizes the effective length of the nonlinear interaction for $\mathrm{THz}$ generation, being essential to use Fourier-limited pump pulses rather than temporally stretched broadband pulses [5]. In addition, maintaining a moderate pumping fluence $\left(\sim 5 \mathrm{~mJ} / \mathrm{cm}^{2}\right)$ ensures no saturation of the $\mathrm{THz}$ generation process due to three-photon absorption [14]. The maximum efficiency predicted at room temperature is approximately 2\% [5]. Assuming a desired output THz energy of $10 \mathrm{~mJ}$, the necessary pump pulse energy is as high as $0.5 \mathrm{~J}$, which implies using a pump spot area of $\sim 100 \mathrm{~cm}^{2}$ or spot diameter of $\sim 11 \mathrm{~cm}$. Currently, it is difficult to grow stoichiometric lithium niobate (sLN) to such size, motivating us to use congruent lithium niobate ( $C L N)$ since it can be produced in much larger sizes, though at a cost of slightly lower conversion efficiency [15]. If the lithium niobate crystal is cooled down to $10 \mathrm{~K}$, pump to $\mathrm{THz}$ conversion efficiencies of up to $13 \%$ have been predicted [5].

In this letter we report on the optimized generation of $\mathrm{THz}$ pulses by optical rectification using pulse front tilting in combination with cryogenically cooled cLN and a close to optimum pump pulse duration of $680 \mathrm{fs}$.

We used a commercial diode-pumped Yb:KYW chirped pulse amplification system (s-Pulse, Amplitude Systemes) capable of generating up to $2 \mathrm{~mJ}$ pulses with 1-kHz repetition rate, $1030 \mathrm{~nm}$ of central wavelength, and $2.6 \mathrm{~nm}$ of spectral bandwidth. To seed the regenerative amplifier, we generated 100 fs, $0.2 \mathrm{~nJ}$ pulses at $80 \mathrm{MHz}$ repetition rate with a stable, mode-locked Yb-doped fiber oscillator [16]. The pulses are stretched to a few ps in a fiber stretcher and pre-amplified in a Yb-doped fiber amplifier to 1.6 $\mathrm{nJ}$ of energy. After the further stretching in a grating stretcher and the regenerative amplification to 2 $\mathrm{mJ}$, the pulses were compressed to $680 \mathrm{fs}, 15 \%$ longer than its transform-limited pulse duration, using a grating compressor. After all optical elements, the maximum available energy was $1.2 \mathrm{~mJ}$ for the experiments. 


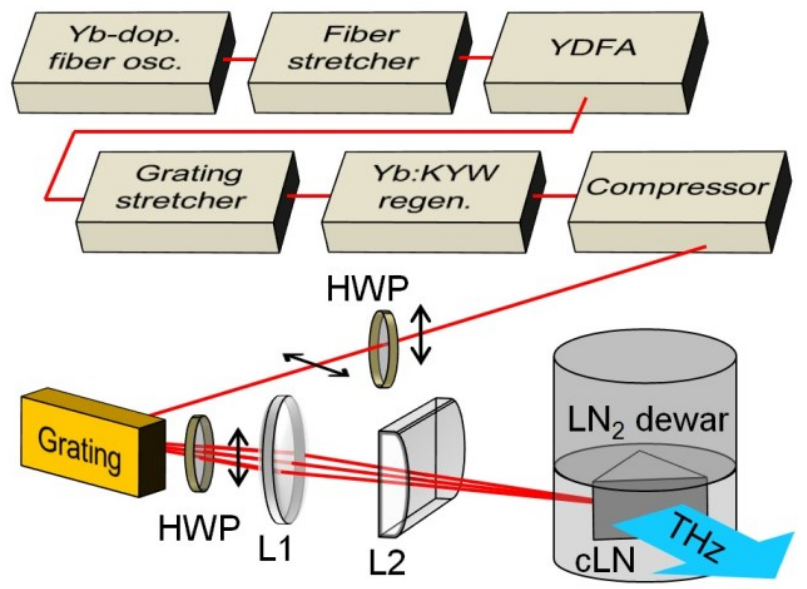

Fig 1. Experimental setup for THz generation. L1: bestform lens with $f=20 \mathrm{~cm} ; \mathrm{L} 2$ : Cylindrical lens with $\mathrm{f}=15 \mathrm{~cm}$; HWP: Half wave plate at $1030 \mathrm{~nm}$.

For $\mathrm{THz}$ generation in the $\mathrm{CLN}$ crystal, a tilted pulse front pumping scheme with optimized parameters was used as described below. The front of the pump pulses was tilted using a 1500 lines $/ \mathrm{mm}$ grating and imaged with a demagnification factor of 1.54 using a $f=20 \mathrm{~cm}$ bestform lens. We selected for $\mathrm{THz}$ generation $\mathrm{CLN}$ prism doped with $\mathrm{MgO}$ at $6.0 \%$, z-cut, cut into an isosceles triangle with an apex angle of 56 degrees (side dimensions of $57.9 \times 57.9 \times 54.4 \mathrm{~mm}^{3}$ and height of $25.4 \mathrm{~mm}$ ). The IR beam experiences total internal reflection inside the lithium niobate, and so only the input and output faces of the crystal are AR coated, whereas the third face remained uncoated. A half waveplate was used to rotate the polarization of the diffracted beam to vertical polarization, parallel to the optic axis of the lithium niobate. To optimize the intensity of the pump beam, we utilized a cylindrical lens that shaped the pump spot size to $3.0 \mathrm{~mm}$ in the horizontal and $3.0 \mathrm{~mm}$ in the vertical direction $\left(1 / \mathrm{e}^{2}\right)$, achieving an optimum fluence of $4.7 \mathrm{~mJ} / \mathrm{cm}^{2}$. The crystal is indium soldered to a nickel plate that matches the thermal expansion coefficient of lithium niobate from room temperature to cryogenic temperatures to ensure no deformations and good thermal contact. The nickel plate is mounted in a commercial cryogenic dewar with monitored temperature. For measuring the produced $\mathrm{THz}$ output we used a calibrated pyroelectric detector (Microtech Instruments) [5], while the repetition rate of the pump laser was decreased to $10 \mathrm{~Hz}$ to avoid saturation in the detector, which is slow. The THz pulse energy was then measured from the voltage modulation observed with a scope, with a sensitivity of $3.4 \mathrm{~V} / \mathrm{mW}$ at $0.5 \mathrm{THz}$. 


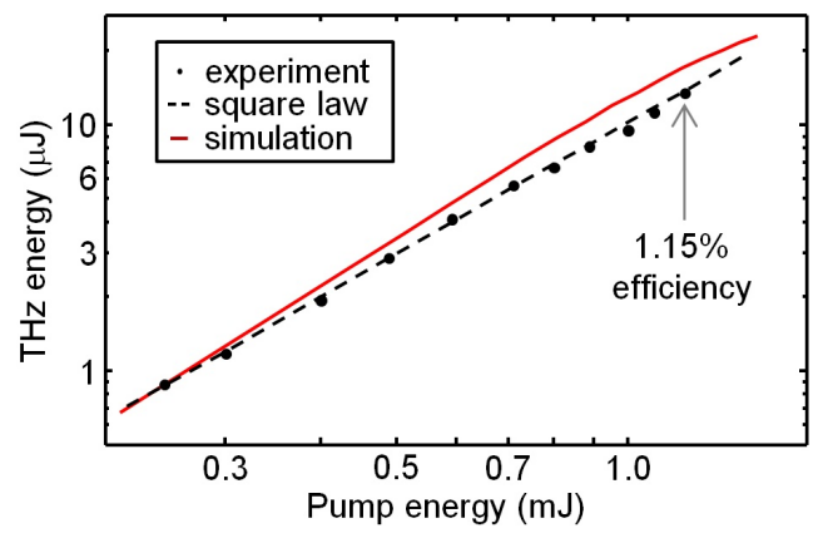

Fig 2. Measured and calculated THz energy versus pump energy at room temperature from CLN.

The measured $\mathrm{THz}$ output energy as a function of pump energy is depicted in Fig 2. The THz energy increases with a power dependence of about 1.8 without any sign of saturation. At the maximum pump energy of $1.2 \mathrm{~mJ}$, the conversion efficiency was about $1.15 \%$, corresponding to $13.5 \mathrm{uJ}$ of THz energy. For the numerical modeling, we solved a one-dimensional equation for the Fourier component of the $\mathrm{THz}$ field followed from Maxwell's equation with a slowly varying envelope approximation, as described in [14]. The theoretical model includes variation of the pump intensity along the propagation distance due to material and angular dispersion, noncollinear propagation direction of IR pump and THz beams, and the absorption of the $\mathrm{THz}$ pulse. We observed a very good match between the theoretical prediction of $1.5 \%$ and the experimental result at room temperature, $1.15 \%$. We suggest that the deviation between the model and experimental results is mainly due to free carrier absorption in the lithium niobate; the $\mathrm{THz}$ absorption increases nonlinearly as the pump energy reaches the $\mathrm{mJ}$ level and thus the efficiency curve starts to show saturation.

Although the pulse shape was not measured, previous works show that the measured THz pulse shape and spectra are in good agreement. For our $680 \mathrm{fs}$ pump pulses, the expected output spectrum is centered at around $0.5 \mathrm{THz}$ [14]. Figure 3 shows the spectral broadening experienced by the IR beam after the $\mathrm{THz}$ generation stage. This is a typical signature of highly efficient $\mathrm{THz}$ generation, where cascaded OR can greatly surpass the Manley-Rowe limit [17]: an excitation photon emitting one $\mathrm{THz}$ photon converts into an optical beam with a small red-shift that can be re-used for $\mathrm{THz}$ generation if the phase matching condition is fulfilled [9]. 


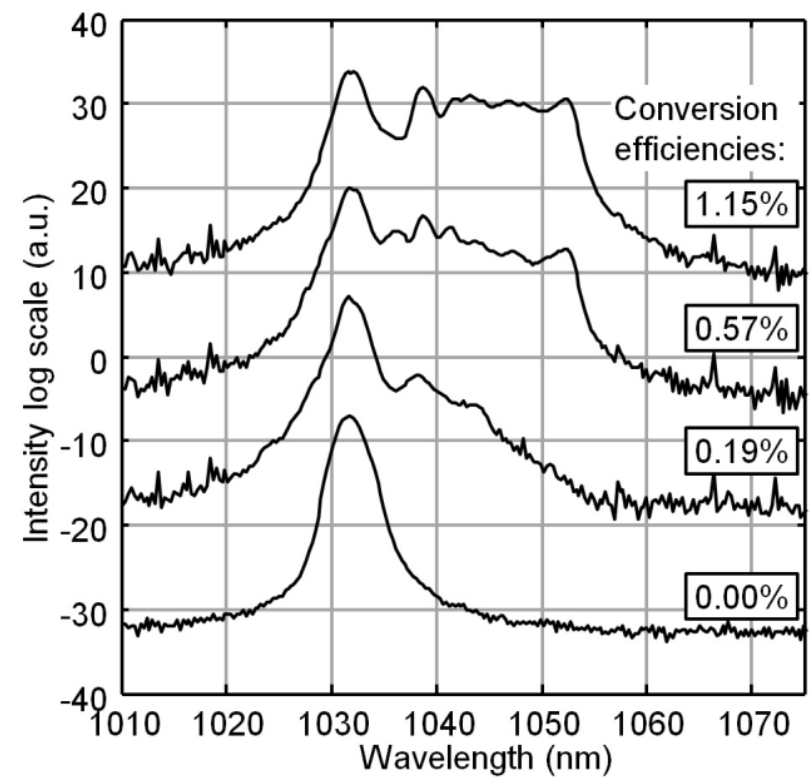

Fig. 3. Spectral broadening of the IR beam after THz generation depending on conversion efficiency.

To further enhance the efficiency, we lowered the absorption of lithium niobate at THz frequencies by cooling it to cryogenic temperatures. As thermal activation is thought to perturb the coherent interaction process, highly efficient generation of $\mathrm{THz}$ waves is expected at low temperatures [18]. The temperature dependence of the $\mathrm{THz}$ power from the lithium niobate crystal at a fixed pump energy of $1.2 \mathrm{~mJ}$ is shown in Fig. 4. The THz power increases monotonically as the temperature decreases to about $150 \mathrm{~K}$. Below this temperature, we observe saturation of the conversion process and even a slight decrease in the detected $\mathrm{THz}$ power, which may have many causes ranging from spectral shifting of the generated $\mathrm{THz}$ radiation and a wavelength dependent detection efficiency or increased free carrier absorption at $\mathrm{THz}$ frequencies. We observed a maximum enhancement of 3.3x, which corresponds to an estimated conversion efficiency of $3.8 \% \pm 0.4 \%$. During the measurements from 280 to $77 \mathrm{~K}$, no optical damage owing to cooling was observed in the crystal. The $\mathrm{THz}$ wavelength is expected to blue-shift slightly due to changes of the refractive index of lithium niobate [18], and also changes the sensitivity of the detector, we estimated a $\pm 0.4 \%$ deviation in the efficiency utilizing the pyroelectric detector calibration curve provided by Microtech Instruments.

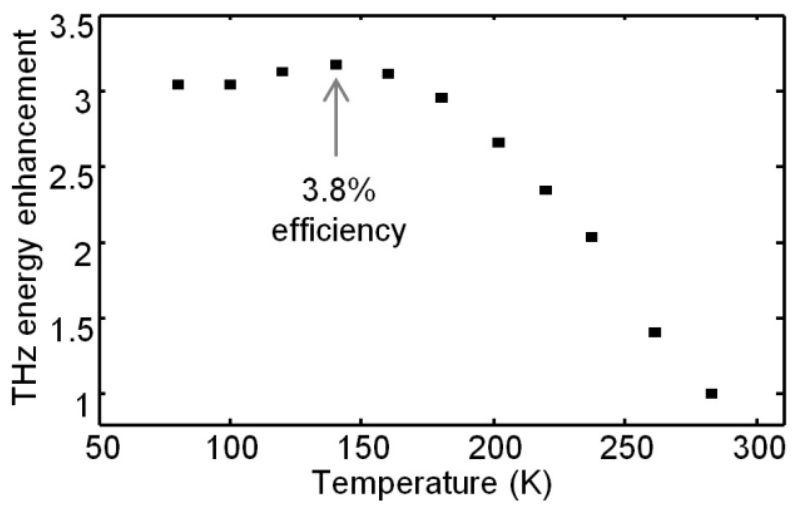


Fig. 4. Efficiency enhancement versus temperature at a fixed pump energy of $1.2 \mathrm{~mJ}$.

In conclusion, we have investigated the conversion efficiency enhancement of $O R$ in lithium niobate by cooling the crystal down to cryogenic temperatures and using near-optimum pump pulses of 0.68 ps. Our $\mathrm{THz}$ source has shown a record conversion efficiency from optical to $\mathrm{THz}$ of $3.8 \%$, close to the maximum theoretical limit ( $6 \%$ for cryogenically cooled cLN at $100 \mathrm{~K}$ ). Our THz source is expected to play an important role in various applications, such as particle acceleration and manipulation. Further study is required to explain the decrease of the output $\mathrm{THz}$ power from $150 \mathrm{~K}$ to $77 \mathrm{~K}$.

The authors acknowledge support by Dr. Daniel Miller from MIT Lincoln Laboratory for mounting of the cryogenically cooled Lithium Niobate crystal. This work was supported by DARPA under contract N66001-1-11-4192. Ronny Huang acknowledges support from a MIT Lincoln Scholar fellowship.

References:

[1] J. Hebling, K.-L. Yeh, M. C. Hoffmann, and K. A. Nelson, "High-power THz generation, THz nonlinear optics, and THz nonlinear spectroscopy," IEEE J. Sel. Top. Quantum Electron. 14, 345-353 (2008).

[2] H. Hirori, M. Nagai, and K. Tanaka, "Excitonic interactions with intense terahertz pulses in ZnSe/ZnMgSSe multiple quantum wells", Phys. Rev. B 81, 081305 (2010).

[3] A. Dienst, M. C. Hoffmann, D. Fausti, J. C. Petersen, S. Pyon, T. Takayama, H. Takagi, and A. Cavalleri, "Bi-directional ultrafast electric-field gating of interlayer charge transport in a cuprate superconductor," Nat. Photon. 5, 485 (2011).

[4] Emeric Balogh, Katalin Kovács, József A. Fülöp, Péter Dombi, Gyozo Farkas, János Hebling, Valer Tosa, and Katalin Varjú, "Single Attosecond Pulse from Terahertz Assisted High-order Harmonic Generation," in CLEO/Europe and EQEC 2011 Conference Digest, OSA Technical Digest (CD) (Optical Society of America, 2011), paper CG_P2.

[5] J. A. Fülöp, L. Pálfalvi, S. Klingebiel, G. Almási, F. Krausz, S. Karsch, and J. Hebling, "Generation of sub$\mathrm{mJ}$ terahertz pulses by optical rectification," Opt. Lett. 37, 557-559 (2012).

[6] A. Sell, A. Leitenstorfer, and R. Huber, "Phase-locked generation and field-resolved detection of widely tunable terahertz pulses with amplitudes exceeding $100 \mathrm{MV} / \mathrm{cm}$," Opt. Lett. 33, 2767-2769 (2008).

[7] M. C. Hoffmann, and J. A. Fülöp, "Intense ultrashort terahertz pulses: generation and applications," J. Phys. D: Appl. Phys. 44083001 (2011).

[8] K. Vodopyanov, "Optical generation of narrow-band terahertz packets in periodically inverted electro-optic crystals: conversion efficiency and optimal laser pulse format," Opt. Express 14, 2263-2276 (2006). 
[9] M. Jewariya, M. Nagai, and K. Tanaka, "Enhancement of terahertz wave generation by cascaded X(2) processes in LiNbO3," J. Opt. Soc. Am. B 26, A101-A106 (2009).

[10] F. Blanchard, L. Razzari, H. Bandulet, G. Sharma, R. Morandotti, J. Kieffer, T. Ozaki, M. Reid, H. Tiedje, H. Haugen, and F. Hegmann, "Generation of $1.5 \mu \mathrm{J}$ single-cycle terahertz pulses by optical rectification from a large aperture ZnTe crystal," Opt. Express 15, 13212-13220 (2007).

[11] J. Hebling, K. Yeh, M. Hoffmann, B. Bartal, and K. Nelson, "Generation of high-power terahertz pulses by tilted-pulse-front excitation and their application possibilities," J. Opt. Soc. Am. B 25, B6-B19 (2008).

[12] József András Fülöp, László Pálfalvi, Matthias C. Hoffmann, and János Hebling, "Towards generation of mJ-level ultrashort THz pulses by optical rectification," Opt. Express 19, 15090-15097 (2011).

[13] L. Palfalvi, J. Hebling, J. Kuhl, A. Peter, and K. Polgar, "Temperature dependence of the absorption and refraction of Mg-doped congruent and stoichiometric LiNbO3 in the THz range," J. Appl. Phys. 97, 123505 (2005)

[14] J. A. Fülöp, L. Pálfalvi, G. Almási, and J. Hebling, "Design of high-energy terahertz sources based on optical rectification," Opt. Express18(12), 12311-12327 (2010).

[15] Zhang Xianbin, Li Yunfeng, Ma lijuan, Yuan ke and Shi Wei, "The impact of MgO-doped nearstoichiometric lithium niobate crystals on the THz wave output characteristics," J. Phys.: Conf. Ser. 276012226 (2011).

[16] K. H. Hong, A. Siddiqui, J. Moses, J. Gopinath, J. Hybl, F. O. Ilday, T.Y. Fan and F. X. Kärtner, "Generation of $287-\mathrm{W}, 5.5-\mathrm{ps}$ pulses at $78-\mathrm{MHz}$ repetition rate from a cryogenically-cooled Yb:YAG amplifier seeded by a fiber chirped-pulse amplification system," Optics Letters 33:(21), pp. 2473-2475 (2008).

[17] K. Vodopyanov, "Optical generation of narrow-band terahertz packets in periodically inverted electro-optic crystals: conversion efficiency and optimal laser pulse format," Opt. Express 14, 2263-2276 (2006).

[18] J. Shikata, M. Sato, T. Taniuchi, H. Ito, and K. Kawase, "Enhancement of terahertz-wave output from LiNbO3 optical parametric oscillators by cryogenic cooling," Opt. Lett. 24, 202-204 (1999). 
